\title{
Drive-by Bridge Damage Monitoring: Concise Review
}

\author{
Ahmed Elhattab* and Nasim Uddin \\ University of Alabama at Birmingham, USA
}

Submission: June 12, 2017; Published: July 07, 2017

*Corresponding author: Ahmed Elhattab, University of Alabama at Birmingham, 1075 13th St S, Birmingham, AL 35205, USA, Tel: 205-447-2127; Email: aahattab@uab.edu

\begin{abstract}
Recently in the area of bridge health monitoring, there is a shift towards the use of indirect measurements of a passing vehicle to assess the structural integrity of bridge structures, which is known as "Drive-by Bridge inspection." This approach is based on determining the dynamic characteristics of the bridge using the vehicle responses, such as bridge frequencies, bridge mode shapes, and even bridge stiffness and damping. The change in these characteristics over a period of time reflects the level of deterioration of the bridge's health condition. This article introduces a brief discussion on the development of this field of research since it was revealed. The article addresses the prominent studies in this domain, while some other work is not included for the sake of brevity in the paper. This article is a good starting point for those interested in researching this topic.
\end{abstract}

Abbreviations: TRLs: Technological Readiness Levels; VBI: Vehicle-Bridge Interaction; ODSC: Operating Deflection Shape Curvature; STFT: Short Time Fourier Transform; FDD: Frequency Domain Decomposition; ESI: Element Stiffness Index

\section{Introduction}

Bridges are amongst the essential elements of transport infrastructure, but are subject to continuous degradation due to environmental effects and may experience increases in traffic weight and volume over time. Therefore, they require continuous monitoring to ensure maintenance and hence their structural integrity. Recent studies firmly established the Technological Readiness Levels (TRLs) of the sensor-based SHM techniques. However, the necessity for power, data storage and transfer and the complexity of the installation hinder successful deployment of the approach to a wide range of bridges [1]. Moreover, this procedure requires a lane closure, which results in a devastating effect on the roadway capacity [2-4] Recently, there has been a paradigm shift in the bridge health monitoring by moving the instrumentation from the bridge body to a transit vehicle, this concept is known as "Drive-by Bridge inspection" [5,6].

This article provides a recapitulation for the work that has been carried out in this field. To establish a consistent review, the article categorizes the proposed approaches under two broad categories, frequency domain analysis, and time domain analysis. The paper highlights the most intellectual work in this field from the authors' perspective.

\section{Frequency Domain Analysis}

\section{Monitoring of the fundamental bridge frequency}

Yang et al. $[7,8]$ were of the first to propose a preliminary study in extracting the fundamental frequency of the bridge from a passing vehicle responses. The authors derived a mathematical formula for the vehicle-bridge interaction problem, showing that the vehicle responses comprise four frequencies which are: the vehicle frequency, the driving speed frequency and two shifted bridge frequencies. This work has established the feasibility of the drive-by concept. Further, the authors investigated the change in the damping ratio of the bridge as a representative of bridge damage. They observed a drop in the shifted bridge frequencies as the bridge damping ratios increases. This work has been asserted in the field by Lin \& Yang [9] where the authors successfully quantified the fundamental frequency of an existing bridge in Taiwan. The authors found that extracting the bridge frequency is attainable for low driving speeds.

The authors interpret this observation to the higher frequency resolution that is obtained at low driving speeds. Furthermore, the car frequencies have a smaller interference in the spectrum when the vehicle slowly moves. On the other hand, the spectrum shows a drastic drop in the signal power, where low speeds lead to a smaller excitation for the bridge. Oshima et al. $[10,11]$ recommended using a vehicle equipped with a shaker device to apply a considerable vibration to the bridge. In a field test, the authors asserted the success of the approach in identifying higher frequency content for the bridge from the vehicle response $[10,11]$. In the same vein, Yang et al. [12] investigated the variation in the fundamental bridge frequencies due to the mass of the inspection vehicle. The authors declare 


\section{Civil Engineering Research Journal}

that this regard it is essential, where the shift induced by the vehicle mass is of the same order as the shift caused by structural degradation.

This observation has been verified using scaled lab test and also in the field by Chang et al. [13]. In two studies made by Fujino et al. [14] and Siringoringo \& Fujino [15], the authors examined identifying the bridge frequency utilizing a periodic monitoring using a light commercial vehicle. The authors tackled the parameters that affect the accuracy of the approach. Their results were similar to previous work; it is recommended to use a low speed to reduce vehicle vibration interference in the response spectrum. The authors also observed a subversive impact from the approach slab and expansion joints on the accuracy of the extracted frequency. These findings deem compelling when they compared to the effect of the roughness on the approach.

The precedent studies demonstrated the concept of extracting the bridge frequencies, indirectly, utilizing a passing vehicle responses. Kim \& Kawatani [5] extended this concept to identify the change in dynamic properties of the bridge due to structural damages. They introduced this concept as "Drive-by Bridge Inspection." They employed the change in the fundamental frequency of the bridge as a measure of damage. Similar work has been carried out by Toshinami et al. [16].

McGetrick et al. [17] pointed out the importance of including the road roughness while investigating the drive-by bridge inspection concept. The authors observed a distinct drop in the acceleration spectra when damage is prevalent if the road profile is smooth. Whereas for the rough profile, the spectra did not show evidence of damage and instead the vehicle frequencies dominate the spectra. Keenahan et al. [18] solved the aforementioned dilemma by introducing the subtraction process for the acceleration signal of two consecutive axles.

Rather than directly processing the acceleration signal, the authors suggested subtracting the acceleration histories of two consecutive axles when they pass the same location ( $x$ ) on the bridge. In another word, for a point located at (x) distance from the bridge start, the acceleration of the front axle when it cross that point is denoted by (af), while the acceleration of the rear axle is denoted by (ar). Subtracting (ar) from (af) is assumed to substantially reduce the effect of the road roughness on the recorded signal. The process is repeated for (n) sections to find the total subtracted signal. The subtracted signal was then processed using FFT to plot the acceleration spectrum. The results exposed a substantial removal of the roughness effect, and damage becomes evident in the spectrum.

Elhattab et al. [19,20] investigated this concept using commercialized FE program (LS-Dyna). The program comprises an explicit solver, which is different from the implicit solver that has been employed in the previous studies to simulate the Vehicle-Bridge Interaction (VBI). The explicit solver performs convergence for each time step, therefore the solution is evolving over the time domain. This module is suitable for highly transit dynamic problems. Whereas the implicit solver seeks convergence over the whole time domain at once, this makes it more suitable for scudo-static problems. The authors simulated the VBI in the LS-Dyna, and the vehicle acceleration was processed using FFT. The authors found the same results when using the spectrum of the subtracted acceleration signal, except they discovered that damage is more evident in the spectrum in this case.

Li W et al. [21] employed an optimization scheme, the Generalized Pattern Search Algorithm (GPSA), to extract the bridge frequency from the vehicle responses. The algorithm successfully determined the fundamental bridge frequency and the bridge stiffness, even in the presence of a very high noise in the signal (SNR=5). Unfortunately, the roughness effect on the approach has not been explored.

\section{Monitoring of the bridge mode shape}

Another trend in the drive-by bridge inspection is to identify the damage using bridge mode shapes. A discontinuity in the mode shape implies a presence of damage in the vicinity of the discontinuity [22-25]. Zhang Y et al. [25] introduced a remarkable study in this field, where they utilized a specialized vehicle named the "tapping vehicle" to extract the bridge mode shape in a field-scale test. The vehicle was equipped with a shaker and was instrumented with accelerometers. The vehicle employed a particular excitation while recording the bridge response to compute the point of impedance. The authors showed that the amplitude of the spectra of the point impedance is proportional to the square of the mode shapes.

Yang Y et al. [26] coined a procedure to estimate the bridge mode shape using instantaneous amplitude of the Hilbert Transform (HT). In the absence of the road roughness, the modes were successfully extracted from the vehicle responses. The authors showed that indirect inspection has a distinct advantage over sensor base inspection, where the vehicle acts as a mobile sensor which collects information for all bridge degrees of freedom. Malekjafarian \& OBrien [27] incorporated the outputonly Frequency Domain Decomposition (FDD) method with the drive-by bridge inspection to develop a new technique known as Short-Time Frequency Domain Decomposition (STFDD).

The author used the signal of two consecutive quarter cars to construct the bridge mode shape. The bridge has been divided into segments and then the local mode of each segment has been extracted using the FDD. Afterward, the local modes have been correlated to construct the global bridge mode shape. The approach has been shown to work properly at low speeds, while the road roughness effect has been resolved using a truck trailer model and the subtraction procedure of Keenahan et al. [18]. 


\section{Civil Engineering Research Journal}

\section{Time Domain Analysis}

\section{Bridge properties}

The precedent work utilized the fundamental frequency and dynamic parameters as a damage index for the bridge health condition. Curadelli et al. [28] introduced the damping as an alternative damage measure. The authors used wavelet transform to establish a procedure identifies the undamped frequency and damping coefficient as a function of time. They experimentally investigated the procedure by testing a reinforced concrete beam, where the damage was imposed by applying a monotonic load to the beam. It has been shown that damping ratio and the damage severity are correlated, whereas the damage increases, the calculated damping coefficient increases too. McGetrick et al. [17] used this finding to study the feasibility of using an instrumented truck to extract informationon bridge condition from changes in its damping value.

The simulation of the vehicle-bridge interaction was performed in MATLAB [17]; the vehicle was represented as a quarter cars and the damage as an increase in bridge damping. They showed that the approach works well in identifying the change in the damping ratio for smooth profiles, while for rough profiles the acceleration spectra are dominated by the vehicle excitation due to the roughness of the road. González et al. [29] build an algorithm that identifies the bridge damping ratio using the axle acceleration history of an instrumented vehicle. The algorithm searches for the damping value that minimizes the error in the back-calculated road profile under the front and rear axles of the vehicle.

The authors have harnessed the same algorithm to estimate average bridge stiffness [30]. Zhang et al. [31] utilized the "tapping vehicle" to extract the Operating Deflection Shape Curvature (ODSC) to reflect the damage severity in the bridge. The Operating Deflection Shape (ODS) is the square of the vehicle frequency obtained by transforming the acceleration to the frequency domain using Short Time Fourier Transform (STFT).While the ODSC is the square root for a normalized ODS [25]. The approach shows to detect local damages accurately considering a smooth intact profile.

\section{Bridge responses}

Kim CW et al. [32] illuminated the drive-by bridge inspection by conducting a scaled test performing three distinct levels of health monitoring using the vehicle measurements. Level I screening was investigating extracting the bridge frequency from the vehicle responses as a measure of damage. This is similar to the work discussed in Section 4 4.1. Alternatively, Level II developed an Auto Regression (AR) model to identify the variation in the dynamic parameters (bridge damping and bridge frequencies) of the bridge using the vehicle responses only. These two screening are considered an output-only screening, where measurements are confined to vehicle measurements only. Level III screening utilizes synchronized measurements from the vehicle and the bridge to estimate the variation of the stiffness along the bridge.

The stiffness was updated by discretizing the bridge equation of motion into a series of equations of single degree of freedom systems. The force on each single degree of freedom equals the force induced by the vehicle, and was calculated using the vehicle responses. While the responses in the equations were the recorded bridge responses along the bridge. Knowing the force and the response, the bridge was divided into segments and the stiffness of each segment was updated over time. The change in the bridge stiffness was represented using the Element Stiffness Index (ESI) which has been coined by the authors. The ESI was calculated as the ratio between the stiffness of the bridge segments to the highest stiffness amongst all segments. These three screening have been explored using a scaled laboratory test.

The bridge was an inverted I-beam, and the damage has been represented as an increase in bridge damping, saw cuts in the beam flange and ridge cut in the beam flange. Level I screening showed similar attribute to the previous studies, where low speed is needed to identify the bridge frequency accurately. This level has been examined to detect damage as an increase in bridge damping ratio. The result shows a drop in the power of the spectrum as the damping increases, however, bridge frequency was not evident in the spectrum and the drop was in the vicinity of the vehicle frequency.

The authors examined Level II screening in detecting the variation in the bridge frequencies (the first and the second fundamental frequencies) and damping ratios due to the adopted damage models. The authors found that for the frequencies, the results are substantially biased. On the other hand, the damping showed a change in its pattern when damping increases; however, the calculated damping was subjected to appreciable error. Lastly, Level III has been investigated to localize the damage and quantify its severity. The proposed method successfully detects the damage location and provides a clue for its severity, in most cases. The proposed method showed to be insensitive to the vehicle type and the vehicle speed. Further, the approach successfully identified multiple damages on the bridge.

Miyamoto \& Yabe [33,34] made a novel achievement in the drive-by bridge inspection approach. They used a public bus instrumented with accelerometers at the front axle to monitor the structural anomaly parameters of the bridge. They estimated the bridge characteristic-deflection using wave integrals obtained using Fourier Transform. The noise influence is reduced by averaging different reading for the same bridge. The results were compared to the readings of accelerometers installed on the bridge, and it showed a good match. The authors showed that damage starts to take place when the calculated characteristic-deflection exceeds specific limits. 


\section{Civil Engineering Research Journal}

Obrien \& Keenahan [35] introduced a novel concept, where they monitor the variation in the bridge displacement due to structural damages using measurements from a passing vehicle. They used the Traffic Speed Deflectometer (TSD) to measure the relative displacement history between the road surface and a horizontal beam on the truck. This displacement history equals the road roughness heights plus the bridge displacement which has been denoted by the authors as the Apparent Profile "AP." If the AP of a damaged bridge is subtracted from the AP of a healthy bridge, the result will be the variation in the bridge displacement due to the existence of the damage. The approach showed to be quite sanative to small damage levels.

Elhattab et al. [36] extended OBrien \& Keenahan [35] work to calculate the AP using non-specialized vehicle instead of using the TSD. The authors suggested using a vehicle instrumented with accelerometers, where the recorded acceleration is used to compute the reaction of the road on the vehicle tires for each time step. The application of these forces on the vehicle tires will mimic the excitation of the vehicle when it passed over the bridge, and though, the displacement history of the contact point will equal the road roughness heights plus the bridge displacement, or the AP. The proposed approach showed to be insensitive to signal noise and to the effect of the initial condition. However, the method is shown to be very sensitive to the wearing of the road roughness over time. The authors suggest that the current profile model is vague noisy and inappropriate to represent the roughness effect for the drive-by bridge inspection studies, and recommended revisiting it.

\section{Conclusion}

The review presents a concise for the most remarkable studies in the drive-by bridge inspection field, which is a good start for those interested in researching this topic. The early work in this domain investigated the feasibility of quantifying the bridge natural frequency from passing vehicle responses. The results reveal the success of determining the fundamental frequency, but only for low speeds. On the other hand, frequency changes due to structural damages tend to be small to reflect the structural health condition of the bridge. From another perspective, the mass of the inspection truck drives a significant shift in the frequency of the bridge, so that the effect of structural damages on the bridge frequency is completely masked because of the impact of this mass.

Another obstacle to this approach has been found, namely, the roughness of the road. Studies have indicated that the roughness of the road excites the vehicle more than the bridge does. Therefore, in the presence of the road roughness in the VBI problem, the vehicle responses overwhelm the acceleration spectrum, whilst fading the bridge frequency. One of the proposed solutions for this particular problem is to subtract the signal of two consecutive axles to substantially remove the road roughness effect on the recorded signal. While the authors herein recommend that it is imperative to re-visit the used roughness model and its plausibility to drive-by bridge inspection application, where it has been developed mainly as a measure of the quality of the roughness of the road, not to mimic the vehicle excitation on the road.

Another group of authors has utilized the mode shapes as a frequency based damage monitoring technique. Damage is remarked as a discontinuity in the bridge mode shape. Similarly, the proposed approaches require low speed to construct the mode shapes properly. Some authors recommended using a specialized inspection truck to stimulate targeted bridge modes. Other authors pointed out to the positive effect for the ongoing traffic on the extracted mode.

In another vein, some studies investigated monitoring the dynamic bridge parameters in the time domain. The most prominent work in this field is the work of Kim CW et al. [32], where they studied both the time and the frequency based approaches. The proposed drive-by method successfully employed the vehicle measurements to tackle the damage; however, the estimated dynamic parameters of the bridge were subjected to an appreciable error. In contrast, when the vehicle and bridge measurements are fused, damages are precisely estimated. This approach worked for single and multiple damages along the bridge. Other studies investigated the average bridge stiffness, bridge damping, and bridge displacement as damage indices. However, regardless the potential of the proposed methods, further studies are needed to shift them from status quo to field-scale testing.

\section{Acknowledgement}

The authors wish to express their gratitude for the financial support received from the National Science of Foundation (NSFCNS-1645863). Any opinions, findings, and conclusions or recommendations expressed in this publication are those of the authors and do not necessarily reflect the view of the sponsors.

\section{References}

1. Malekjafarian A, McGetrick PJ, OBrien EJ (2015) A review of indirect bridge monitoring using passing vehicles. Shock and Vibration 2015: 286139.

2. Ramadan O, Sisiopiku V (2016) Impact of Bottleneck Merge Control Strategies on Freeway Level of Service. Transportation Research Procedia 15: 583-593.

3. Ramadan OE, Sisiopiku VP (2015) Bottleneck Merge Control Strategies for Work Zones: Available Options and Current Practices. Open Journal of Civil Engineering 5(4): 428-436.

4. Ramadan OE, Sisiopiku VP (2016) Evaluation of merge control strategies at interstate work zones under peak and off-peak traffic conditions. Journal of transportation technologies 6(03): 118-130.

5. Kim CW, Kawatani M (2009) Challenge for a drive-by bridge inspection. Osaka, Japan.

6. Kim CW, Isemoto R, Toshinami T, Kawatani M, McGetrick PJ, et al. (2011) Experimental investigation of drive-by bridge inspection. Cancun, Mexico. 


\section{Civil Engineering Research Journal}

7. Yang Y, Lin C (2005) Vehicle-bridge interaction dynamics and potential applications. Journal of sound and vibration 284(1): 205-226.

8. Yang YB, Lin C, Yau J (2004) Extracting bridge frequencies from the dynamic response of a passing vehicle. Journal of Sound and Vibration 272(3): 471-493.

9. Lin C, Yang Y (2005) Use of a passing vehicle to scan the fundamental bridge frequencies: An experimental verification. Engineering Structures 27(13): 1865-1878.

10. Oshima Y, Yamaguchi T, Kobayashi Y, Sugiura K (2008) Eigenfrequency estimation for bridges using the response of a passing vehicle with excitation system. doi.org/10.1201/9781439828434.ch376.

11. Oshima Y, Yamamoto K, Sugiura K, Yamaguchi T (2009) Estimation of bridge eigenfrequencies based on vehicle responses using ICA. Osaka, Japan.

12. Yang Y, Cheng M, Chang K (2013) Frequency variation in vehicle-bridge interaction systems. Int J Str Stability Dyn 13(02): 1350019.

13. Chang K, Kim C, Borjigin S (2014) Variability in bridge frequency induced by a parked vehicle. Smart Structures and Systems 13(5): 755-773.

14. Fujino Y, Kitagawa K, Furukawa T, Ishii H (2005) Development of vehicle intelligent monitoring system (VIMS). SPIE Proceedings $\mathrm{p}$. 5765.

15. Siringoringo DM, Fujino Y (2012) Estimating bridge fundamental frequency from vibration response of instrumented passing vehicle: Analytical and experimental study. Advances in Structural Engineering 15(3): 417-433.

16. Toshinami T, Kawatani M, Kim C (2010) Feasibility investigation for identifying bridge's fundamental frequencies from vehicle vibrations. CRC Press, Boca Raton, Florida, USA.

17. McGetrick PJ, González A, OBrien EJ (2009) Theoretical investigation of the use of a moving vehicle to identify bridge dynamic parameters. Insight-Non-Destructive Testing and Condition Monitoring 51(8): 433438

18. Keenahan J, OBrien EJ, McGetrick PJ, González A (2013) The use of a dynamic truck-trailer drive-by system to monitor bridge damping. Structural Health Monitoring 13(2): 143-157.

19. El-hattab A, Uddin N, Obrien E (2015) Drive-by bridge damage detection using apparent profile. First International Conference on Advances in Civil Infrastructure and Construction Materials 2015: 3345 .

20. ElHattab A, Uddin N, OBrien E (2017) Drive-by bridge damage detection using non-specialized instrumented vehicle. Bridge Structures 12(34): 73-84.

21. Li W, Jiang Z, Wang T, Zhu H (2014) Optimization method based on Generalized Pattern Search Algorithm to identify bridge parameters indirectly by a passing vehicle. Journal of Sound and Vibration 333(2): 364-380.
22. Pandey A, Biswas M, Samman M (1991) Damage detection from changes in curvature mode shapes. Journal of sound and vibration 145(2): 321-332.

23.Zhu X, Law S (2006) Wavelet-based crack identification of bridge beam from operational deflection time history. International Journal of Solids and Structures 43(7): 2299-22317.

24. Arora V, Singh S, Kundra T (2009) Damped model updating using complex updating parameters. Journal of Sound and Vibration 320(1): 438-451.

25. Zhang Y, Wang L, Xiang Z (2012) Damage detection by mode shape squares extracted from a passing vehicle. Journal of Sound and Vibration 331(2): 291-307.

26. Yang Y, Li Y, Chang K (2014) Constructing the mode shapes of a bridge from a passing vehicle: a theoretical study. Smart Structures and Systems 13(5): 797-819.

27. Malekjafarian A, OBrien EJ (2014) Identification of bridge mode shapes using short time frequency domain decomposition of the responses measured in a passing vehicle. Engineering Structures 81: 386-397.

28. Curadelli R, Riera J, Ambrosini D, Amani M (2008) Damage detection by means of structural damping identification. Engineering Structures 30(12): 3497-3504.

29. González A, OBrien EJ, McGetrick PJ (2012) Identification of damping in a bridge using a moving instrumented vehicle. Journal of Sound and Vibration 331(18): 4115-4 31.

30. González A, OBrien EJ, McGetrick PJ (2010) Detection of bridge dynamic parameters using an instrumented vehicle. $5^{\text {th }}$ World Conference on Structural Control and Monitoring, Tokyo, Japan.

31. Zhang Y, Lie ST, Xiang Z (2013) Damage detection method based on operating deflection shape curvature extracted from dynamic response of a passing vehicle. Mechanical Systems and Signal Processing 35(1): 238-254.

32. Kim CW, Isemoto R, McGetrick P, Kawatani M, O’Brien EJ (2014) Driveby bridge inspection from three different approaches Smart Structures and Systems 13(5): 775-796.

33. Miyamoto A, Yabe A (2011) Bridge condition assessment based on vibration responses of passenger vehicle. Journal of Physics: Conference Series 305 (2011): 012103.

34. Miyamoto A, Yabe A (2012) Development of practical health monitoring system for short-and medium-span bridges based on vibration responses of city bus. Journal of Civil Structural Health Monitoring 2(1): 47-63.

35. OBrien EJ, Keenahan J (2015) Drive-by damage detection in bridges using the apparent profile. Structural Control and Health Monitoring 22(5): 813-825.

36. Elhattab A, Uddin N, OBrien E (2016) Drive-by bridge damage monitoring using Bridge Displacement Profile Difference. Journal of Civil Structural Health Monitoring 6(5): 839-850. 


\section{Your next submission with Juniper Publishers} will reach you the below assets

- Quality Editorial service

- Swift Peer Review

- Reprints availability

- E-prints Service

- Manuscript Podcast for convenient understanding

- Global attainment for your research

- Manuscript accessibility in different formats

( Pdf, E-pub, Full Text, Audio)

- Unceasing customer service

Track the below URL for one-step submission https://juniperpublishers.com/online-submission.php 